\title{
Chronic progressive external ophthalmoplegia in a Saudi patient with a mutation in the POLG gene successfully managed with bilateral frontalis sling
}

\author{
Hussein Algahtani ${ }^{1, *(\infty)}$, Bader Shirah ${ }^{2, \pm \oplus}$, Khalid Alsaggaf ${ }^{3 \oplus}$, Mohammad H. Al-Qahtani $^{4 \oplus}$, Angham Abdulrahman Abdulkareem ${ }^{4 \oplus}$, \\ Muhammad Imran Naseer, ${ }^{4,5}$, and Ahmad R. Abuzinadah ${ }^{6 \oplus}$ \\ ${ }^{1}$ Neurology Section, Department of Medicine, King Abdulaziz Medical City, King Saud bin Abdulaziz University for Health Sciences, Jeddah, \\ Saudi Arabia \\ ${ }^{2}$ King Abdullah International Medical Research Center, King Saud bin Abdulaziz University for Health Sciences, Jeddah, Saudi Arabia \\ ${ }^{3}$ College of Medicine, King Saud bin Abdulaziz University for Health Sciences, Jeddah, Saudi Arabia \\ ${ }^{4}$ Center of Excellence in Genomic Medicine Research, King Abdulaziz University, Jeddah, Saudi Arabia \\ ${ }^{5}$ Department of Medical Laboratory Technology, Faculty of Applied Medical Sciences, King Abdulaziz University, Jeddah, Saudi Arabia \\ ${ }^{6}$ Division of Neurology, Department of Internal Medicine, King Abdulaziz University Hospital, College of Medicine, King Abdulaziz University, \\ Jeddah, Saudi Arabia
}

Chronic progressive external ophthalmoplegia (CPEO) is a complex slowly progressive mitochondrial disorder characterized by extraocular muscle weakness with or without multisystem involvement. The mainstay of therapy in a patient with CPEO is supportive. However, in moderate cases, surgery might be indicated including surgeries for ptosis and strabismus. In this article, we report a Saudi patient with CPEO due to compound heterozygous variants in the DNA polymerase gamma $(P O L G)$ gene c.2246T>C p.(Phe749Ser) and c.1735C >T p.(Arg579Trp), which are classified as pathogenic. Proper diagnosis with genetic testing confirmation is important to guide the management and counsel the patient about the prognosis and the management options. The patient was successfully managed with bilateral frontalis sling and illustrates the importance of surgical intervention to improve vision and cosmetic appearance in patients with CPEO. We emphasize the importance of multidisciplinary care in the management of cases of mitochondriopathy, especially CPEO.

Key words: DNA polymerase gamma, Chronic progressive external ophthalmoplegia, Ophthalmologic surgical procedures, Saudi Arabia.

\section{Introduction}

Chronic progressive external ophthalmoplegia (CPEO) is a complex slowly progressive mitochondrial disorder character- ized by extraocular muscle weakness with or without multisystem involvement [1]. It is a rare genetic disorder commonly caused by a single mitochondrial deoxyribonucleic acid (DNA) deletion and is considered the most frequent manifestation of

Received: 3 June 2021, Revised: 18 August 2021, Accepted: 20 August 2021, Published: 31 December 2021

${ }^{*}$ Corresponding author: Hussein Algahtani, M.D., FRCPC, MMed. (iD https://orcid.org/0000-0001-9484-9838

Neurology Section, Department of Medicine, King Abdulaziz Medical City, King Saud bin Abdulaziz University for Health Sciences, Jeddah 21483, Saudi Arabia.

Tel: +96-65-5663-3130, E-mail: halgahtani@hotmail.com

${ }^{\ddagger}$ Current affiliation: Department of Neuroscience, King Faisal Specialist Hospital \& Research Centre, Jeddah, Saudi Arabia

Conflict of interest: The authors declare that they do not have any conflicts of interest.

(c) This is an open-access article distributed under the terms of the Creative Commons Attribution Non-Commercial License (http://creativecommons.org/licenses/by-nc/4.0/) which permits unrestricted non-commercial use, distribution, and reproduction in any medium, provided the original work is properly cited.

(c) Copyright 2021 by the Korean Society of Medical Genetics and Genomics 

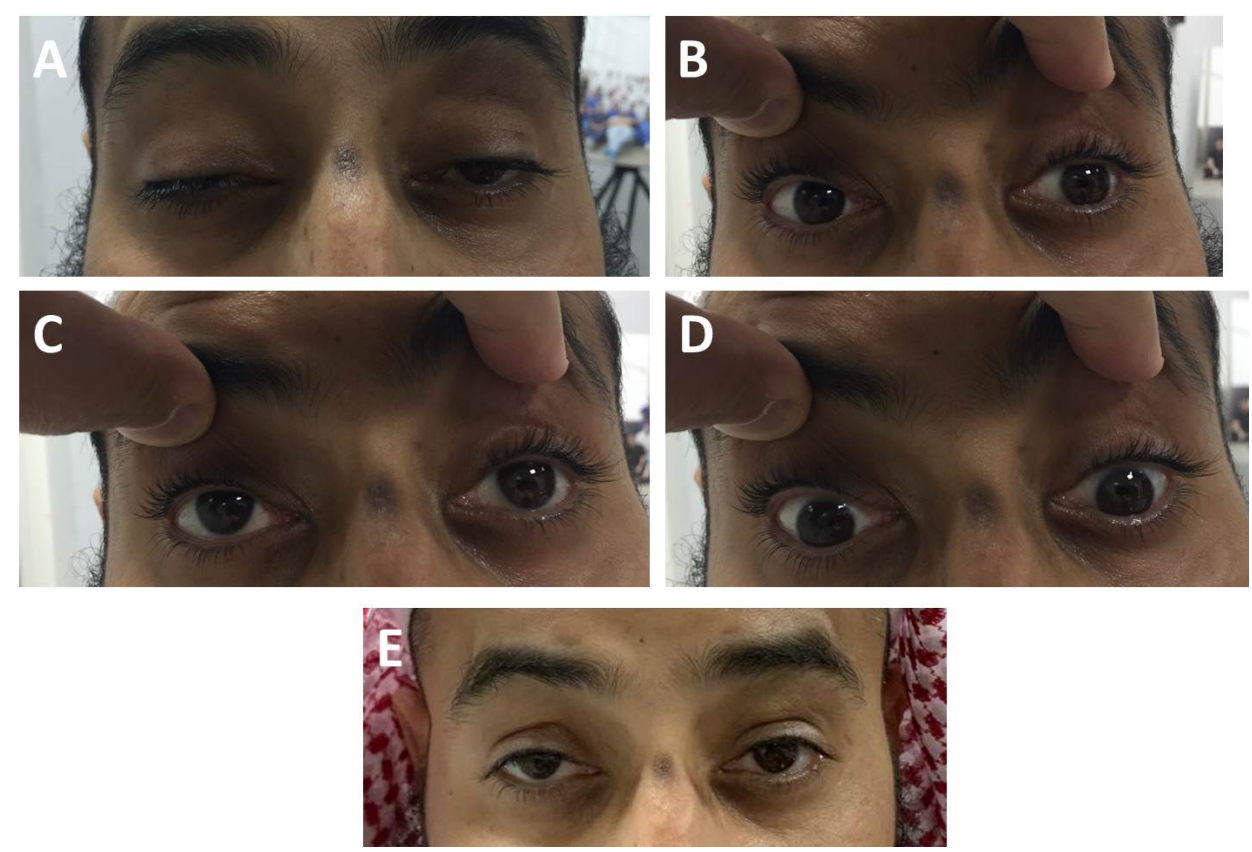

Fig. 1. Bilateral ptosis and ocular movements examination showing severe external ophthalmoplegia and improvement after surgery. (A) Dextroversion, (B) levoversion, (C) supraversion, (D) infraversion, and $(E)$ six months postoperative image.

mitochondrial myopathies. It is attributed to sporadic mutations, nuclear-inherited mutations, and maternally inherited mutations of mitochondrial DNA [2]. Up to $90 \%$ of patients with mitochondrial myopathies have ocular abnormalities, and CPEO is the presenting feature of two-thirds of the cases. It causes dysfunction of extraocular muscles, skeletal muscles, retina, cochlea, heart, and brain [3]. Ptosis is a prominent and usually severe component of CPEO. Although extraocular muscle motility dysfunction is often symmetrical, an asymmetric presentation may occur. There is no diurnal variation or exacerbation with fatigue of either the extraocular motility dysfunction or ptosis. Visual acuity is typically spared, and diplopia is a rare occurrence [4]. The mainstay of therapy in a patient with CPEO is supportive. However, in moderate cases, surgery might be indicated including surgeries for ptosis and strabismus. Additionally, in severe cases, a surgery called bilateral frontalis sling could be performed [5]. In this article, we report a Saudi patient with CPEO due to compound heterozygous variants in the DNA polymerase gamma $(P O L G)$ gene successfully managed with bilateral frontalis sling. The written informed consent was obtained from the patient.

\section{Case}

A 38-year-old male presented to the neurology clinic complaining of bilateral drooping of the eyelids of 8 years duration. At the initial presentation, ptosis started and was noticed first in the right eye then the left eye was affected a few months later.
The eye symptoms worsened in the last three years with no clear fluctuation. He also reported intermittent diplopia and slurring of speech, and his speech became difficult to understand. He had muscle cramps at both rest and activity that started in the right leg then spread to all four limbs with worsening during cold exposure. He had a fatigable weakness as he needs to take a rest after short distance walking. There were no sensory, cardiac, autonomic, or constitutional symptoms. He is known to have diabetes mellitus since he was 16 years old. There is no family history of a similar condition or any other neurological disorder. The ptosis and ophthalmoplegia did not improve with a six months trial of prednisone and pyridostigmine.

On examination, he had bilateral ptosis with restriction of extraocular muscle movement (ophthalmoplegia) (Fig. 1). The fundus examination revealed a normal retina, and his visual acuity was normal with eyeglass correction. Motor examination showed normal power in the upper and lower extremities with absent reflexes bilaterally. The sensory and cerebellar examinations were normal. We confirm that a written informed consent was obtained from the patient.

General and biochemical blood tests were unremarkable apart from elevated blood glucose and hemoglobin A1C. Nerve conduction study and electromyography showed electrodiagnostic evidence of sensory neuronopathy with no myopathic motor unit potentials. The muscle biopsy showed mild chronic inflammatory cells and myopathic changes in skeletal muscle fibers but did not reveal features of mitochondrial myopathies. Magnetic resonance imaging of the brain and echocardiogra- 
A

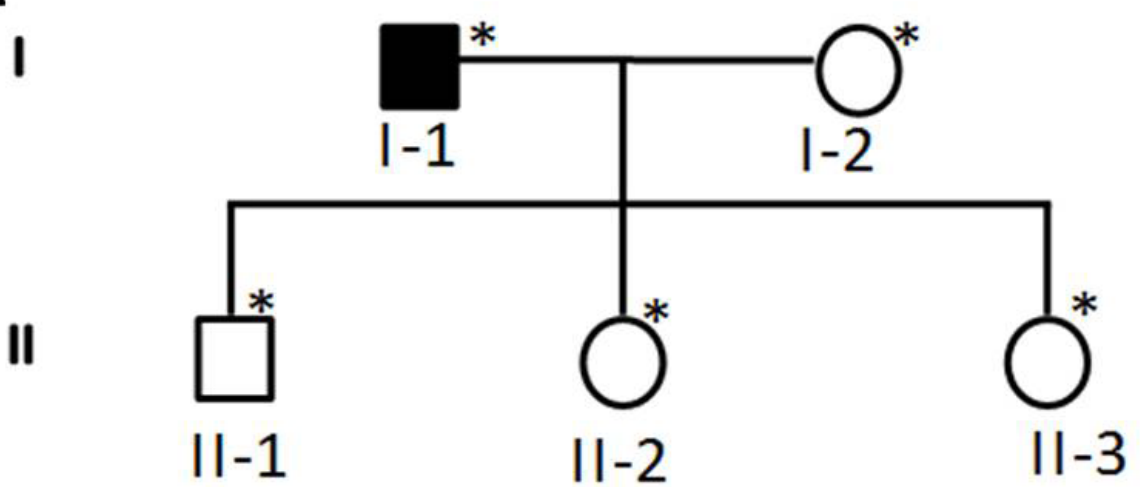

B

I-1

$1-2$

II-1

$\|-2$

II-3

Control
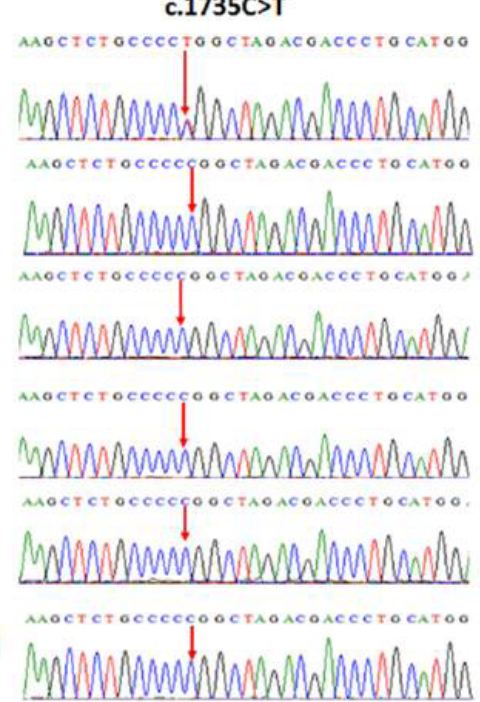

POLG

$1-2$

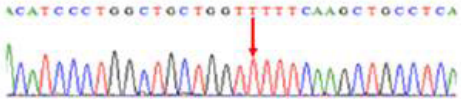

II-1

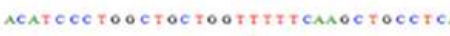

II-2

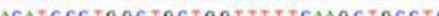

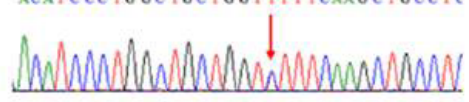

II-3

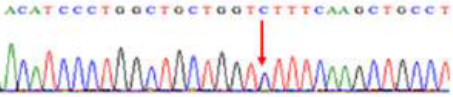

Control

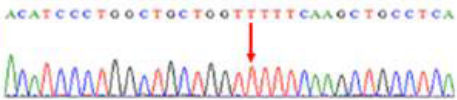

Fig. 2. (A) Family pedigree showing the details of the members of the family. The samples marked with asterisks were available for genetic testing. (B) Representative chromograph of DNA polymerase gamma (POLG) Sanger sequencing read of the available family members. Genetic analysis identified this variant C.1735C $>$ T only in the affected patient (I-1), while c.2246T $>C$ variant was present in the patient (I-1) as well as in his asymptomatic offspring (II-1, $\|-2\|-3$,$) .$ phy were normal. Whole exome sequencing was performed and revealed two heterozygous variants in the POLG gene c.2246T>C p.(Phe749Ser) and c.1735C>T p.(Arg579Trp), which are classified as pathogenic (Fig. 2). Considering the consistent phenotype of the patient and compound heterozygosity of the two pathogenic variants in $P O L G$, a genetic diagnosis of CPEO is confirmed. In addition, Sanger sequencing was performed for the asymptomatic offspring of the patient to identify the affected individuals (Fig. 2).

His symptoms were bothersome, and he elected to go for surgery for the ptosis. Bilateral frontalis sling was performed, and the patient's visual abilities and self-esteem were significantly improved after surgery (Fig. 1).

\section{Discussion}

Mitochondria are cytoplasmic organelles and the energy producer in the cell with dual genetic control mechanisms since their function is controlled by genes in both mitochondrial DNA and nuclear DNA. More than 37 mitochondrial genes and 1,500 mitochondrial proteins have been described [6]. Mitochondriopathies are a group of genetic disorders of children and adults with heterogeneous and diverse clinical presentations making the diagnosis a real challenge. Although many cases of mitochondriopathies may not be diagnosed, the estimated worldwide prevalence ranges between 1/5,000 and 1/8,500. Severe phenotypes present early in the course of the disease even in the neonatal period, while milder ones manifest in the third and fourth decade of life and even later [7]. The mitochondrial disorders that are associated with ptosis, ophthalmoplegia, and myopathy phenotypes include CPEO, myoclonic epilepsy with ragged-red fibers, Kearns-Sayre syndrome, sensory ataxia neuropathy, dysarthria, ophthalmoplegia, and myopathy, neurogastrointestinal encephalopathy [8]. 
CPEO is a common manifestation of mitochondriopathy, which may overshadow neurological involvement or involvement of other skeletal muscles. Clinically, it usually presents between the ages of 18 and 40 years with characteristic eye manifestations including ptosis, extraocular muscle weakness, and ophthalmoplegia. There are multiple forms of inheritance of CPEO including sporadic, autosomal dominant, autosomal recessive, and maternal. Moreover, the defect in DNA could be a result of point mutations. However, the majority of cases develop the disease by large-scale mitochondrial DNA rearrangement. Mutations in POLG1,POLG2, SPG7,DGUOK, and $R R M 2 B$ genes are linked to the development of the autosomal recessive and autosomal dominant types of CPEO due to defects in intergenomic signaling resulting in multiple mitochondrial DNA deletions [9]. Systemic manifestations such as generalized myopathy, axonal neuropathy, ataxia, depression, hypogonadism, Parkinsonism, and cataracts could be seen in patients with autosomal dominant CPEO due to POLG mutation. On the other hand, POLG mutation leading to autosomal recessive CPEO is not associated with systemic manifestations [10]. Our patient's phenotype of ptosis, diplopia, slurring of speech, muscle cramps, and fatigable weakness was consistent with mitochondrial myopathy despite the absence of mitochondrial changes on muscle biopsy. The presence of sensory neuronopathy was beyond what is expected from diabetes and suggested that a mitochondrial condition is more likely. Considering the consistent phenotype of the patient and compound heterozygosity of the two pathogenic variants in $P O L G$, a genetic diagnosis of CPEO was confirmed.

The mechanism underpinning the selective involvement of extraocular muscles with resultant characteristic clinical features is poorly understood. Several theories were proposed by conducting ultrastructural and biochemical studies. The fundamentally distinct structural, functional, biochemical, and immunological properties of extraocular muscles compared to other skeletal muscles include higher mitochondrial content, higher blood flow, higher motor neuron discharge rate, smaller motor unit size, and higher reactive oxygen species production [11]. In addition, patients with CPEO with mitochondrial DNA deletion had more cyclooxygenase (COX)-deficient fibers in extraocular muscles as compared to skeletal muscles. A less severe form of the disease is seen in patients with mitochondrial DNA point mutation [12]. Further collaborative studies may further clarify these mechanisms.

The diagnosis of CPEO is challenging, and the differential diagnosis includes myasthenia gravis, ocular myositis, thyroid- associated orbitopathy, oculopharyngeal muscular dystrophy, and congenital fibrosis of the extraocular muscles. The diagnostic workup, which definitely confirms the diagnosis of CPEO, is a molecular genetic study in addition to muscle biopsy, which is used when the molecular genetic study cannot confirm the diagnosis. In addition, further investigations for mimickers may be warranted [13]. In our patient, the diagnosis of myasthenia gravis was applied, and the patient was started on anticholinesterase inhibitors treatment with no benefit. Important features to differentiate between CPEO and myasthenia gravis include fatigability, fluctuation of clinical features, diurnal variation, involvement of other muscle groups such as bulbar muscles, facial muscles, and limb muscles. In addition, serological testing for acetylcholine receptor antibody and muscle-specific tyrosine kinase antibody are further differentiating tools.

Muscle biopsies in patients with CPEO typically show a subsarcolemmal accumulation of ragged-red fibers (abnormal mitochondria) and a mosaic pattern of COX-deficient fibers with abnormal COX activity. The abnormal mitochondrial changes occur when the accumulation of mutated mitochondrial DNA exceeds a biochemical threshold (threshold effect) [14]. In our patient, a muscle biopsy was normal with a lack of ragged-red fibers, which are considered to be the morphological hallmark of mitochondrial myopathy. Performing muscle biopsy is a pathological challenge since the standardization of the process is mandatory, and protocols to preserve morphology, enzymatic activity, and genetic content should be utilized. A multidisciplinary approach including surgeons, neurologists, and pathologists, should be required to achieve the goals of muscle biopsy. The results of limb muscle biopsy in patients with CPEO are diagnostic in around $75 \%$ of cases. DNA analysis is required to investigate the remaining cases [15].

Peripheral neuropathy is a typical finding in patients with CPEO. However, it is variably expressed based on the genetic abnormality. It is more common in multiple deletions of mitochondrial DNA than in single deletions. Electrophysiological studies in patients suffering from mitochondrial disorders usually reveal a predominantly axonal type of nerve fiber damage. Other forms including a predominantly demyelinating pattern or a mixed axonal and demyelinating type of peripheral nerve damage were also reported [16]. In our patient, the neurophysiological testing revealed a pure severe sensory axonal neuronopathy with minimal sensory symptoms. The presence of sensory neuronopathy is a very important clue to guide the genetic testing as it is found more with nuclear-encoded mitochondriopathy such as POLG gene mutation more than mitochondrial DNA-related 
mitochondriopathoes $[17,18]$. Electrophysiological evaluation in patients with CPEO should be a routine investigation to document the systemic nature of the disease.

Patients with CPEO suffer from a high frequency of severe fatigue, depression, pain, and impairment of activities of daily living with dependence on others for activities of daily life. The frequency and severity of these symptoms are higher in CPEO patients suffering from diabetes as compared to non-diabetics. In addition, the cosmetic effect and facial appearance of patients with CPEO may contribute to depression [19]. Ptosis may be corrected by surgery, as seen in our patient, with a significant impact on these psychosocial complications. Long-term follow-up is required as patients may progressively lose their ability to close their eyelids and develop exposure keratopathy [20].

In conclusion, we reported a rare case of CPEO in a Saudi patient who was successfully managed with bilateral frontalis sling. Proper diagnosis with genetic testing confirmation is important to guide the management and counsel the patient about the prognosis and the management options. The present case illustrates the importance of surgical intervention to improve vision and cosmetic appearance in patients with CPEO. We emphasize the importance of multidisciplinary care in the management of cases of mitochondriopathy, especially CPEO.

\section{Authors' Contributions}

Conception and design: HA, BS. Acquisition of data: BS, KA, MIN. Analysis and interpretation of data: HA, BS, MHA, AAA, MIN, ARA. Drafting the article: HA, BS, KA. Critical revision of the article: MHA, AAA, MIN, ARA. Final approval of the version to be published: all authors.

\section{References}

1. Wong $\sqcup$, Naviaux RK, Brunetti-Pierri N, Zhang Q, Schmitt ES, Truong C, et al. Molecular and clinical genetics of mitochondrial diseases due to POLG mutations. Hum Mutat 2008;29:E150-72.

2. Stumpf JD, Saneto RP, Copeland WC. Clinical and molecular features of POLG-related mitochondrial disease. Cold Spring Harb Perspect Biol 2013;5:a011395.

3. Finsterer J, Zarrouk-Mahjoub S, Daruich A. The eye on mitochondrial disorders. J Child Neurol 2016;31:652-62.

4. Yu-Wai-Man C, Smith FE, Firbank MJ, Guthrie G, Guthrie S, Gorman GS, et al. Extraocular muscle atrophy and central nervous system involvement in chronic progressive external ophthalmoplegia. PLoS One 2013;8:e75048.

5. Rajabi MT, Tabatabaie SZ, Rajabi MB, Abrishami Y, Hosseini SS, Oestreicher J. Management of myogenic ptosis in chronic progressive external ophtalmoplegia. Iran J Neurol 2014;13:185-7.

6. Bastian $A E$, Jugulete $G$, Manole $E$, Oprişan LA. A rare case of mitochondriopathy with autosomal dominant progressive external ophthalmoplegia diagnosed through skeletal muscle biopsy. Rom J Morphol Embryol 2019;60:273-9.

7. Chinnery PF. Primary mitochondrial disorders overview. In: Adam MP, Ardinger $\mathrm{HH}$, Pagon RA, eds. GeneReviews ${ }^{\circledR}$. Seattle (WA): University of Washington, Seattle, 1993-2020.

8. Angelini $C$, Bello L, Spinazzi M, Ferrati C. Mitochondrial disorders of the nuclear genome. Acta Myol 2009;28:16-23.

9. McClelland C, Manousakis G, Lee MS. Progressive external ophthalmoplegia. Curr Neurol Neurosci Rep 2016;16:53.

10. Rahman S, Copeland WC. POLG-related disorders and their neurological manifestations. Nat Rev Neurol 2019;15:40-52.

11. Yu Wai Man CY, Chinnery PF, Griffiths PG. Extraocular muscles have fundamentally distinct properties that make them selectively vulnerable to certain disorders. Neuromuscul Disord 2005;15:17-23.

12. Greaves LC, Yu-Wai-Man P, Blakely EL, Krishnan KJ, Beadle NE, Kerin J, et al. Mitochondrial DNA defects and selective extraocular muscle involvement in CPEO. Invest Ophthalmol Vis Sci 2010;51:3340-6.

13. Schoser BG, Pongratz D. Extraocular mitochondrial myopathies and their differential diagnoses. Strabismus 2006;14:107-13.

14. Bacalhau $M$, Simões $M$, Rocha $M C$, Hardy $S A$, Vincent $A E$, Durães J, et al. Disclosing the functional changes of two genetic alterations in a patient with chronic progressive external ophthalmoplegia: report of the novel mtDNA m.7486G>A variant. Neuromuscul Disord 2018;28:350-60

15. Sundaram $C$, Meena $A K$, Uppin MS, Govindaraj $P$, Vanniarajan $A$ Thangaraj $K_{1}$ et al. Contribution of muscle biopsy and genetics to the diagnosis of chronic progressive external opthalmoplegia of mitochondrial origin. J Clin Neurosci 2011;18:535-8.

16. Lehmann D, Kornhuber ME, Clajus C, Alston CL, Wienke A, Deschauer $\mathrm{M}$, et al. Peripheral neuropathy in patients with CPEO associated with single and multiple mtDNA deletions. Neurol Genet 2016;2:e113.

17. Lax NZ, Whittaker RG, Hepplewhite PD, Reeve AK, Blakely EL, Jaros E et al. Sensory neuronopathy in patients harbouring recessive polymerase $\gamma$ mutations. Brain 2012;135(Pt 1):62-71.

18. Horga A, Pitceathly RD, Blake JC, Woodward CE, Zapater P, Fratter C, et al. Peripheral neuropathy predicts nuclear gene defect in patients with mitochondrial ophthalmoplegia. Brain 2014;137(Pt 12):3200-12.

19. Smits BW, Fermont J, Delnooz CC, Kalkman JS, Bleijenberg G, van Engelen BG. Disease impact in chronic progressive external oph- 
thalmoplegia: more than meets the eye. Neuromuscul Disord $2011 ; 21: 272-8$.

20. Cohen BH, Chinnery PF, Copeland WC. POLG-related disorders. In:
Adam MP, Ardinger HH, Pagon RA, eds. GeneReviews ${ }^{\circledR}$. Seattle (WA): University of Washington, Seattle, 1993-2021. 